\title{
Downregulation of miR-519a Predicts Poor Prognosis and Contributes to Tumor Progression in Gastric Cancer
}

Huimei Cai ${ }^{a}$ Hui Lin ${ }^{a}$ Wenyu Cao ${ }^{a}$ Juan Sun ${ }^{a}$ Yudian Huang ${ }^{b}$ Yuehua Fang ${ }^{\mathrm{C}}$

${ }^{a}$ Department of Gastroenterology, Fuzhou First Hospital Affiliated to Fujian Medical University, Fuzhou, China; ${ }^{b}$ Department of Pathology, Fuzhou First Hospital Affiliated to Fujian Medical University, Fuzhou, China;

'Department of Clinical Laboratory, Fuzhou First Hospital Affiliated to Fujian Medical University, Fuzhou, China

\section{Retraction Statement See Next Page}




\title{
Retraction Statement
}

\author{
Paper by Huimei Cai, Hui Lin, Wenyu Cao, Juan \\ Sun, Yudian Huang, and Yuehua Fang entitled \\ “Downregulation of miR-519a Predicts Poor \\ Prognosis and Contributes to Tumor Progression \\ in Gastric Cancer" [Oncol Res Treat. 2020;43:19-26, \\ doi: 10.1159/000504054]
}

\begin{abstract}
The article by Huimei Cai, Hui Lin, Wenyu Cao, Juan Sun, Yudian Huang, and Yuehua Fang entitled "Downregulation of miR-519a Predicts Poor Prognosis and Contributes to Tumor Progression in Gastric Cancer" [Oncol Res Treat. 2020;43:19-26, doi: $10.1159 / 000504054$ ] has been retracted by the Publisher and the Editors. Following publication it was brought to our attention that the article had been simultaneously published in another journal by the authors. The authors have not responded to our correspondence regarding this retraction despite multiple attempts of contact.
\end{abstract}

